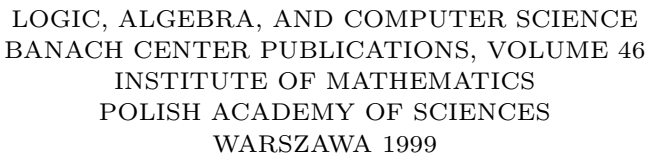

\title{
INTERRELATION OF ALGEBRAIC, SEMANTICAL AND LOGICAL PROPERTIES FOR SUPERINTUITIONISTIC AND MODAL LOGICS
}

\author{
LARISA MAKSIMOVA \\ Institute of Mathematics \\ Siberian Division of Russian Academy of Sciences \\ 630090, Novosibirsk, Russia \\ E-mail:lmaksi@math.nsc.ru
}

We consider the families $\mathcal{L}$ of propositional superintuitionistic logics (s.i.l.) and $N E(\mathbf{K})$ of normal modal logics (n.m.l.). It is well known that there is a duality between $\mathcal{L}$ and the lattice of varieties of pseudo-boolean algebras (or Heyting algebras), and also $N E(\mathbf{K})$ is dually isomorphic to the lattice of varieties of modal algebras. Many important properties of logics, for instance, Craig's interpolation property (CIP), the disjunction property (DP), the Beth property (BP), Hallden-completeness (HP) etc. have suitable properties of varieties as their images, and many natural algebraic properties are in accordance with natural properties of logics. For example, a s.i.l. $L$ has CIP iff its associated variety $V(L)$ has the amalgamation property (AP); $L$ is Hallden-complete iff $V(L)$ is generated by a subdirectly irreducible Heyting algebra. For any n.m.l. $L$, the amalgamation property of $V(L)$ is equivalent to a weaker version of the interpolation property for $L$, and the superamalgamation property is equivalent to CIP; $L$ is Hallden-complete iff $V(L)$ satisfies a strong version of the joint embedding property.

Well-known relational Kripke semantics for the intuitionistic and modal logics seems to be a more natural interpretation than the algebraic one. The categories of Kripke frames may, in a sense, be considered as subcategories of varieties of Heyting or modal algebras. We discuss the question to what extent one may reduce problems on properties of logics to consideration of their semantic models.

1. Preliminaries. A language of propositional modal logic contains the connective $\rightarrow$ and the propositional constant $\perp$, and an unary modal operation $\square$. Other connectives

1991 Mathematics Subject Classification: 03B45, 03F55.

Partially supported by State Committee of Russian Federation for High Education, grant on Informatics for 1996-1997.

The paper is in final form and no version of it will be published elsewhere. 
are defined in the usual way. Denote $[0] A \rightleftharpoons A,[n+1] A \rightleftharpoons A \& \square[n] A$ for each natural number $n$.

If $\mathbf{p}$ is a list of variables, let $F(\mathbf{p})$ denote a formula, such that any its variable is contained in $\mathbf{p}$. The set of all such formulas is denoted by $\mathcal{F}(\mathbf{p})$ and any its subset by $\Gamma(\mathbf{p})$.

By a normal modal logic we mean any set of formulae that contains all the tautologies of two-valued logic and the formula $\square(A \rightarrow B) \rightarrow(\square A \rightarrow \square B)$, and is closed with respect to the substitution rule and also the rules $\mathrm{R} 1: A,(A \rightarrow B) / B$ and $\mathrm{R} 2: A / \square A$. The least normal modal logic is denoted by $\mathbf{K}$. For any logic $L$, let $N E(L)$ denote the set of all normal modal logics containing $L$.

For $L$ in $N E(\mathbf{K})$, we sometimes write $L \vdash A$ instead of $A \in L$; the notation $\Gamma \vdash_{L} A$ means that the formula $A$ is deducible from $\Gamma \cup L$ with help of the rules R1 and R2; we write $\Gamma \vdash_{L}$, if $\Gamma \vdash_{L} A$ for each formula $A$. The least normal extension of $L$ containing a set $A x$ of formulas is denoted by $L+A x$. As usual, we denote $\mathbf{K} \mathbf{4}=\mathbf{K}+(\square p \rightarrow \square \square p)$, $\mathbf{S 4}=\mathbf{K} \mathbf{4}+(\square p \rightarrow p)$.

The deduction theorem holds in every normal modal logic $L$ in the following form:

For any set $\Gamma$ of formulas and for any formulas $A$ and $B$, if $\Gamma, A \vdash_{L} B$ then there exists $n$ such that $\Gamma \vdash_{L}[n] A \rightarrow B$.

With every logic $L$ in $N E(\mathbf{K})$, one can associate in a one-to-one manner a variety of so-called modal algebras. A modal algebra is an algebra $\mathbf{A}=(A, \rightarrow, \neg, \square)$ which satisfies the identities of Boolean algebras for $\rightarrow$ and $\neg$ and, moreover, the conditions $\square \top=\top$ and $\square(x \rightarrow y) \leq \square x \rightarrow \square y$. If $A$ is a formula, then $\mathbf{A} \models A$ denotes that the identity $A=\top$ holds in $\mathbf{A}$. Write $\mathbf{A} \models L$ instead of $(\forall A \in L)(\mathbf{A} \models A)$. Let $V(L)=\{\mathbf{A} \mid \mathbf{A} \models L\}$. One can prove that $L=\{A \mid(\forall \mathbf{A} \in V(L)) \mathbf{A}=A\}$.

The logic $L$ is tabular if $V(L)$ is generated by finitely many finite algebras; $L$ is locally tabular if $V(L)$ is locally finite.

All varieties of modal algebras possess such important properties as the congruence distributivity and the congruence extension property CEP.

An algebra is finitely indecomposable if it cannot be represented as a subdirect product of finitely many proper quotient-algebras. An algebra $\mathbf{A}$ is subdirectly irreducible if it cannot be represented as a subdirect product of algebras different from A. A modal algebra is subdirectly irreducible if and only if it contains a critical element [19]. An element $b$ is critical, whenever $b \neq \top$ and for every $x \neq \top$ there exists an $n$ such that $[n] x \leq b$.

2. Interpolation, amalgamation and the Beth Property. It was proved in [13] that interpolation properties of normal modal logics are closely connected with amalgamation properties of associated varieties of modal algebras. These connections are presented in the diagram below.

In this diagram the following definitions are used.

For our language containing the logical constants $\top$ and $\perp$, the Craig Interpolation Property (CIP) for a logic $L$ is formulated as follows:

If $(A \rightarrow B)$ is in $L$, then there exists a formula $C$ such that $(A \rightarrow C)$ and $(C \rightarrow B)$ are in $L$ and all the variables of $C$ are in both $A$ and $B$. 


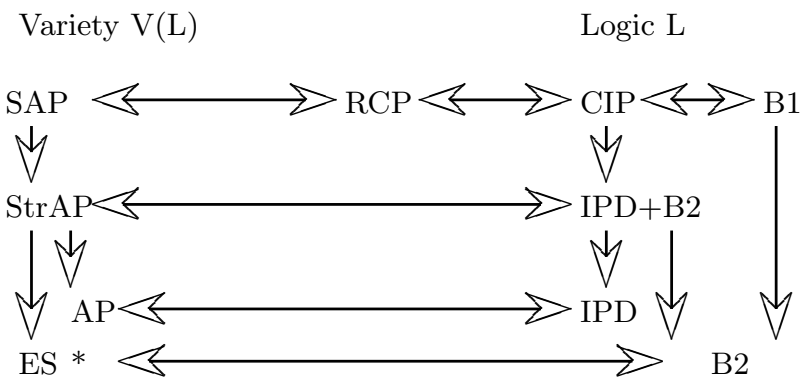

It can be easily shown that in $N E(\mathbf{K}) \mathrm{CIP}$ is equivalent to the Robinson Consistency Principle RCP that is a natural analog of Robinson's Joint Consistency Theorem.

Further, we say that a logic $L$ satisfies the Interpolation Principle for Deducibility (IPD) if the following holds: For any $A$ and $B$ the condition $A \vdash_{L} B$ implies $A \vdash_{L} C$ and $C \vdash_{L} B$ for some formula $C$ such that all the variables of $C$ are in both $A$ and $B$.

Let us fix a list $\mathbf{p}$ of variables which does not contain the variables $x$ and $y$ and define two versions of the Beth Property. We say that a logic $L$ has the Property B1, if the condition $L \vdash A(\mathbf{p}, x) \& A(\mathbf{p}, y) \rightarrow(x \leftrightarrow y)$ implies $L \vdash A(\mathbf{p}, x) \rightarrow(x \leftrightarrow B(\mathbf{p}))$ for some formula $B(\mathbf{p})$.

We get another definition taking the deducibility sign $\vdash_{L}$ instead of implication. Then the Property B2 for a logic $L$ is defined as follows: If $A(\mathbf{p}, x), A(\mathbf{p}, y) \vdash_{L}(x \leftrightarrow y)$ then there exists $B(\mathbf{p})$ such that $A(\mathbf{p}, x) \vdash_{L}(x \leftrightarrow B(\mathbf{p}))$.

Due to the deduction theorem, one can easily prove that CIP implies IPD and B1 implies B2. As it was shown in [14], in modal logics the Property B1 is equivalent to the Craig Interpolation Property that is satisfied rather seldom [8]. The properties IPD and B2 are independent in $N E(\mathbf{K})$ [13]. At the same time, all logics in $N E(\mathbf{K} 4)$ possess B2 [15]. A survey of results on interpolation in normal modal logics is given in [12].

By [13], the Beth Property B2 in a normal modal logic $L$ is equivalent to

$E S^{*}$ : for any $\mathbf{A}, \mathbf{B}$ in $V(L)$, for any monomorphism $\alpha: \mathbf{A} \rightarrow \mathbf{B}$ and for any $x \in$ $\mathbf{B}-\alpha(\mathbf{A})$, such that $\{x\} \cup \alpha(\mathbf{A})$ generates $\mathbf{B}$, there exist $\mathbf{C} \in V$ and monomorphisms $\beta: \mathbf{B} \rightarrow \mathbf{C}, \gamma: \mathbf{B} \rightarrow \mathbf{C}$, such that $\beta \alpha=\gamma \alpha$ and $\beta(x) \neq \gamma(x)$.

As one can see from the diagram, the interpolation properties are closely connected with amalgamation.

Say that a class $K$ has the Amalgamation Property if it satisfies the following condition for any algebras $\mathbf{A}, \mathbf{B}, \mathbf{C}$ in $K$ :

$(A P)$ for any monomorphisms $\beta: \mathbf{A} \rightarrow \mathbf{B}$ and $\gamma: \mathbf{A} \rightarrow \mathbf{C}$ there exists an algebra $\mathbf{D}$ in $K$ and monomorphisms $\delta: \mathbf{B} \rightarrow \mathbf{D}, \epsilon: \mathbf{C} \rightarrow \mathbf{D}$ such that $\delta \beta=\epsilon \gamma$.

If, in addition, $\delta(\mathbf{B}) \cap \epsilon(\mathbf{C})=\delta \beta(\mathbf{A})$ holds, then the class $K$ is said to have the Strong Amalgamation Property $(\operatorname{Str} A P)$.

Finally, $K$ is said to have the Super-Amalgamation Property $(S A P)$ if for any algebras $\mathbf{A}, \mathbf{B}, \mathbf{C}$ in $K$ the condition $A P$ is satisfied and, moreover, for any $x \in \mathbf{B}, y \in \mathbf{C}$ the following equivalences are fulfilled:

$$
\delta(x) \leq \epsilon(y) \Longleftrightarrow(\exists z \in \mathbf{A})(x \leq \beta(z) \& \gamma(z) \leq y)
$$


and

$$
\delta(x) \geq \epsilon(y) \Longleftrightarrow(\exists z \in \mathbf{A})(x \geq \beta(z) \& \gamma(z) \geq y) .
$$

Now we consider a problem: how to prove or disprove amalgamation property for a concrete variety $V$. For this purpose we may reduce our task to consideration of some subclass of $V$ as follows. For a given variety $V$ we denote by $\operatorname{FGFI}(V)$ its subclass of all finitely generated and finitely indecomposable algebras in $V$. The following theorems are especially useful in the case when a logic $L$ is tabular or locally tabular. If $L$ is locally tabular, FGFI $(V(L)))$ contains only finite algebras, and this class is finite, whenever $L$ is tabular. The following statements are proved in [13].

Theorem 2.1. For any logic $L$ in $N E(\mathbf{K})$, the following are equivalent:

(i) $L$ possesses CIP,

(ii) $V(L)$ has the superamalgamation property,

(iii) for any $\mathbf{A}, \mathbf{B}, \mathbf{C}$ in $F G F I(V(L))$, for any monomorphisms $\beta$ : $\mathbf{A} \rightarrow \mathbf{B}, \gamma: \mathbf{A} \rightarrow \mathbf{C}$ and for any $x \in \mathbf{B}, y \in \mathbf{C}$, if $\neg(\exists z \in \mathbf{A})(x \leq \beta(z) \& \gamma(z) \leq y)$ then there exist an algebra $\mathbf{D}$ in $F G F I(V(L))$ and homomorphisms $\delta: \mathbf{B} \rightarrow \mathbf{D}, \epsilon: \mathbf{C} \rightarrow \mathbf{D}$ such that $\delta \beta=\epsilon \gamma$ and not $\delta(x) \leq \epsilon(y)$.

TheOREM 2.2. For any $L$ in $N E(\mathbf{K})$, the following are equivalent:

(i) $L$ possesses IPD,

(ii) $V(L)$ has the amalgamation property,

(iii) for any $\mathbf{A}, \mathbf{B}, \mathbf{C}$ in $F G F I(V(L))$ and for any monomorphisms $\beta$ : $\mathbf{A} \rightarrow \mathbf{B}, \gamma$ : $\mathbf{A} \rightarrow \mathbf{C}$, and for any $y \in \mathbf{C}$, if $y \neq \top$ then there exist an algebra $\mathbf{D}$ in $F G F I(V(L))$ and homomorphisms $\delta: \mathbf{B} \rightarrow \mathbf{D}, \epsilon: \mathbf{C} \rightarrow \mathbf{D}$ such that $\delta \beta=\epsilon \gamma$ and $\epsilon(y) \neq \top$.

TheOREM 2.3. For any $L$ in $N E(\mathbf{K})$ the following are equivalent:

(i) $L$ possesses B2,

(ii) $V(L)$ satisfies $E S^{*}$,

(iii) for any $\mathbf{A}, \mathbf{B}$ in $F G F I(V(L))$, any monomorphism $\alpha: \mathbf{A} \rightarrow \mathbf{B}$ and any $x \in$ $\mathbf{B}-\alpha(\mathbf{A})$, such that $\{x\} \cup \alpha(\mathbf{A})$ generates $\mathbf{B}$, there exist $\mathbf{C}$ in $F G F I(V(L))$ and homomorphisms $\beta: \mathbf{B} \rightarrow \mathbf{C}, \gamma: \mathbf{B} \rightarrow \mathbf{C}$ such that $\beta \alpha=\gamma \alpha$ and $\beta(x) \neq \gamma(x)$.

3. Hallden-Completeness and Joint Embedding Property. We say that a logic $L$ is Hallden-complete (or has the Hallden Property) if for any formulas $A$ and $B$ which have no variables in common the condition $(A \vee B) \in L$ implies that $A \in L$ or $B \in L$.

In [16] the algebraic equivalents of the Hallden-Completeness and of some related property were found.

A class $V$ of algebras is said to have the Joint Embedding Property $(J E P)$ if for any algebras $\mathbf{A}$ and $\mathbf{B}$ in $V$ there exist $\mathbf{C} \in V$ and monomorphisms $\alpha: \mathbf{A} \rightarrow \mathbf{C}, \beta: \mathbf{B} \rightarrow \mathbf{C}$. We say that $V$ has Super-Embedding Property $(J S E P)$ if for any $\mathbf{A}$ and $\mathbf{B}$ in $V$ there exist $\mathbf{C} \in V$ and monomorphisms $\alpha: \mathbf{A} \rightarrow \mathbf{C}, \beta: \mathbf{B} \rightarrow \mathbf{C}$ such that for any $a \in \mathbf{A}-\{\perp\}$ and $b \in \mathbf{B}-\{T\}$ the inequality $\alpha(a) \leq \beta(b)$ does not hold.

Remark. No non-trivial variety of modal algebras possesses the Joint Embedding Property since the degenerate (i.e. one-element) modal algebra is not embeddable into 
a non-degenerate one. If a variety $V$ of modal algebras has the Amalgamation Property then the class of all non-degenerate algebras of $V$ has the Joint Embedding Property, whenever its 0-generated non-degenerate algebra is unique.

Note that each variety $V$ may be completely characterized by its subclass $\operatorname{FGFI}(V)$. We may prove here the theorems which are similar to theorems 2.1 and 2.2. But for our embedding properties we may restrict ourselves to a smaller subclass of $V(L)$.

TheOREm 3.1 [16]. Let $L$ be a normal modal logic, $V(L)$ the associated variety of modal algebras. Then the following are equivalent:

(i) $L$ is Hallden-complete,

(ii) the class of all non-degenerate algebras of $V(L)$ has Super-Embedding Property,

(iii) for any subdirectly irreducible algebras $\mathbf{A}$ and $\mathbf{B}$ in $V(L)$ and for any critical elements $a \in \mathbf{A}, b \in \mathbf{B}$ there exist a subdirectly irreducible algebra $\mathbf{C} \in V(L)$ and monomorphisms $\alpha$ from $\mathbf{A}$ into $\mathbf{C}$ and $\beta$ from $\mathbf{B}$ into $\mathbf{C}$ such that $\alpha(a) \vee \beta(b) \neq \top$.

It is obvious that the condition (iii) implies the Joint Embedding Property of the class of subdirectly irreducible algebras in $V(L)$ :

(iv) for any subdirectly irreducible algebras $\mathbf{A}, \mathbf{B}$ in $V(L)$ there exist a subdirectly irreducible $\mathbf{C}$ in $V(L)$ and monomorphisms from $\mathbf{A}$ into $\mathbf{C}$ and from $\mathbf{B}$ into $\mathbf{C}$.

In general, (iii) is not equivalent to (iv) (see [16]) but (iii) and (iv) are equivalent for logics in $N E(\mathbf{K 4})$.

It appears that the Joint Embedding Property has a natural equivalent in modal logics, too. We say that a logic $L$ possesses the Pseudo-Relevance Property (PRP), if for any formulas $A$ and $B$ without common variables the condition $A \vdash_{L} B$ implies $A \vdash_{L}$ or $\vdash_{L} B$.

Pseudo-Relevance Property was considered in [21] for superintuitionistic logics (in slightly different form). The following theorem was proved in [16].

THEOREM 3.2. Let $L$ be a normal modal logic, $V(L)$ associated variety of modal algebras. Then the following are equivalent:

(i) L has Pseudo-Relevance Property,

(ii) the class of non-degenerate modal algebras of $V(L)$ has Joint Embedding Property,

(iii) any two subdirectly irreducible algebras of $V(L)$ are jointly embeddable into a suitable algebra in $V(L)$.

One can easily prove that in $N E(\mathbf{K})$ the Pseudo-Relevance Property is implied by the Hallden-Completeness but the converse is not true.

In view of connections of implication with disjunction, we might consider the HalldenCompleteness as a special case of the Craig Interpolation Property, when the premise and the conclusion of the implication formula have no variable in common. But it should be noted that it is true only when there are no logical constants different from $\perp$ and $T$. Such a situation holds for normal modal logics containing the logic $\mathbf{D}=\mathbf{K}+\{\diamond \top\}$ or the logic $\mathbf{K}+\square \perp$. In $N E(\mathbf{D})$ the Craig Interpolation Property implies the Hallden-Completeness, and IPD implies PRP. In $N E(\mathbf{K})$ neither CIP nor IPD imply PRP. It is easy to show that $\diamond \top \in L$ or $\square \perp \in L$ for each normal modal logic $L$ with PRP. It follows that if $L$ in 
$N E(\mathbf{K})$ has PRP and CIP then $L$ is Hallden-complete. It is shown in [16] that there is a logic in $N E(\mathbf{D})$ which has IPD and PRP but does not satisfy the Hallden Property.

4. Superintuitionistic logics. It was proved in [7] that there exist exactly seven consistent superintuitionistic logics with the Craig Interpolation Property. It is evident that CIP is equivalent to IPD for superintuitionistic logics. For a superintuitionistic logic $L$, by $V(L)$ we denote the variety of pseudo-Boolean algebras [18] satisfying the identity $A=\top$ for every $A$ in $L$. It was proved in [7] that analogies of theorems 2.1 and 2.2 hold for every superintuitionistic logic. So $S A P, S \operatorname{tr} A P$ and $A P$ are equivalent in the case of varieties of pseudo-Boolean algebras.

Every superintuitionistic logic satisfies the Beth Property [6] and the Pseudo-Relevance Principle [5]. Analogs of theorems 2.3 and 3.2 can also be proved for every superintuitionistic logic. As a corollary, all varieties of pseudo-Boolean algebras have $E S^{*}$ and $J E P$.

An algebraic characterization of superintuitionistic logics which are Hallden-complete was found by A. Wroński [24]. A superintuitionistic logic $L$ is Hallden-complete if and only if its corresponding variety $V(L)$ is generated by a subdirectly irreducible pseudo-Boolean algebra.

It is well-known that superintuitionistic logics are in close relation with normal extensions of the modal logic $\mathbf{S} 4$ (see, for instance, $[2,17]$ ). It is evident that the Halldencompleteness of a modal logic $L$ extending $\mathbf{S} \mathbf{4}$ implies the Hallden-completeness of the superintuitionistic fragment of $L$. It was noted in [1] that some stronger property, namely, so-called Principle of Variable Separation holds in a superintuitionistic logic $L$, whenever some modal companion of $L$ is Hallden-complete. Now we find an algebraic equivalent of the Principle of Variable Separation in superintuitionistic logic.

We say that the Principle of Variable Separation (PVS) holds in a logic $L$, if for any formulas $A_{1}(\mathbf{p}), A_{2}(\mathbf{p}), B_{1}(\mathbf{q}), B_{2}(\mathbf{q})$, where $\mathbf{p}$ and $\mathbf{q}$ are disjoint lists of variables, the condition $L \vdash A_{1}(\mathbf{p}) \& B_{1}(\mathbf{q}) \rightarrow A_{2}(\mathbf{p}) \vee B_{2}(\mathbf{q})$ implies $L \vdash A_{1}(\mathbf{p}) \rightarrow A_{2}(\mathbf{p})$ or $L \vdash B_{1}(\mathbf{q}) \rightarrow B_{2}(\mathbf{q})$.

It is obvious that the Principle of Variable Separation and Hallden-completeness are equivalent for modal logics. It was proved in [9] that CIP implies PVS for any superintuitionistic logic.

The equivalence of (i) and (ii) of theorem 3.1 remains true for $L$ being a superintuitionistic logic and $V(L)$ the corresponding variety of pseudo-Boolean algebras. The condition (iii) may be replaced by (iv) as follows.

Theorem 4.1. Let $L$ be a superintuitionistic logic. Then the Principle of Variable Separation holds in $L$ if and only if for any subdirectly irreducible pseudo-Boolean algebras $\mathbf{A}, \mathbf{B}$ in $V(L)$ there exist a subdirectly irreducible $\mathbf{C}$ in $V(L)$ and monomorphisms from $\mathbf{A}$ into $\mathbf{C}$ and from $\mathbf{B}$ into $\mathbf{C}$.

Now we consider the Disjunction Property DP. We say that a logic $L$ has the Disjunction Property if $(A \vee B) \in L$ implies $A \in L$ or $B \in L$ for any formulas $A, B$.

In $[11]$ the following statement was proved. 
TheOREm 4.2. Let an intermediate logic $L$ be complete with respect a class $K$ of pseudo-Boolean algebras. Then the following are equivalent:

(i) L has the Disjunction Property,

(ii) for any $\mathbf{A}$ and $\mathbf{B}$ in $K$ there exist a $\mathbf{C}$ in $F I(V(L))$ and a homomorphism from $\mathbf{C}$ onto the direct product $\mathbf{A} \times \mathbf{B}$.

It was proved in [1] that the Disjunction Property and the Principle of Variable Separation are independent in the class of superintuitionistic logics.

5. Relational semantics. One can reduce modal and superintuitionistic logics to the corresponding varieties of modal and pseudo-Boolean algebras. The relational Kripke semantics for these logics seems more natural but there are logics which are not Kripkecomplete. The connection between the algebraic and relational semantics is based on representation theorems which were proved for distributive lattices by Birkhoff, for intuitionistic logic by Stone and for Boolean algebras with operators by Jonsson and Tarski.

As usual, a Kripke frame for a modal logic is a pair $\mathbf{W}=<W, R>$, where $W$ is a non-empty set and $R$ a relation on $W$. With any frame $\mathbf{W}=<W, R>$ one can associate a modal algebra $\left.\mathbf{W}^{+}=<W^{+}, \rightarrow, \perp, \square\right)$, where $W^{+}$is the power-set of $W$ and $\perp=\emptyset$, $X \rightarrow Y=(W-X) \cup Y, \square X=\{x \in W \mid \forall y(x R y \Rightarrow y \in X)\}$ for $X, Y \subseteq W$.

For any frame $\mathbf{W}=<W, R>$, say that a subset $X$ of $W$ is a cone if it satisfies the condition: $(x \in X$ and $x R y) \Rightarrow y \in X$. For any $a$ in $W$ let $W^{a}$ be the least cone of $W$ containing $a$, and $\mathbf{W}^{a}=<W^{a}, R \mid W^{a}>$. If $W=W^{a}$, the element $a$ is called an initial of $\mathbf{W}$, and $\mathbf{W}$ is called an initial frame. One can prove that an algebra $\mathbf{W}^{+}$is subdirectly irreducible if and only if $\mathbf{W}$ is an initial frame.

Let $\mathbf{W}=<W, R>$ and $\mathbf{W}^{\prime}=<W^{\prime}, R^{\prime}>$ be any frames, $\theta: W \rightarrow W^{\prime}$ a mapping from $W$ onto $W^{\prime}$. Say that $\theta$ is a p-morphism ([20]) if it satisfies the conditions:

(p1) $w R v \Rightarrow \theta(w) R^{\prime} \theta(v)$ for all $w, v$ in $W$,

(p2) $\theta(w) R^{\prime} v \Rightarrow\left(\exists w^{\prime} \in W\right)\left(w R w^{\prime}\right.$ and $\left.\theta\left(w^{\prime}\right)=v\right)$.

Say that $\mathbf{W}^{\prime}=<W^{\prime}, R^{\prime}>$ is the direct union of a family $\mathbf{W}_{i}=<W_{i}, R_{i}>, i \in I$ of disjoint frames, whenever $W^{\prime}=\bigcup\left\{W_{i} \mid i \in I\right\}, R^{\prime}=\bigcup\left\{R_{i} \mid i \in I\right\}$.

The following lemma can be easily proved.

Lemma 5.1. Let $\mathbf{W}=<W, R>$ and $\mathbf{W}^{\prime}=<W^{\prime}, R^{\prime}>$ be any frames. Then

(i) if $\mathbf{W}^{\prime}$ is a cone of $\mathbf{W}$, then $h(X)=X \cap W^{\prime}$, where $X \subseteq W$, is a homomorphism from $\mathbf{W}^{+}$onto $\mathbf{W}^{\prime+}$;

(ii) if $\theta: W \rightarrow W^{\prime}$ is a p-morphism from $\mathbf{W}$ onto $\mathbf{W}^{\prime}$, then $f(X)=\theta^{-1}(X)$, where $X \subseteq W$, is a monomorphism from $\mathbf{W}^{\prime+}$ into $\mathbf{W}^{+}$;

(iii) if $\mathbf{W} \cup \mathbf{W}^{\prime}$ is the direct union of $\mathbf{W}$ and $\mathbf{W}^{\prime}$ then $\left(\mathbf{W} \cup \mathbf{W}^{\prime}\right)^{+}$is isomorphic to the direct product $\mathbf{W}^{+} \times \mathbf{W}^{\prime+}$.

Now consider a frame $\mathbf{W}=<W, R>$, where $R$ is reflexive and transitive. Denote by $B(W)$ the set of all cones of $\mathbf{W}$, let $\top=W, X \& Y=X \cap Y, X \vee Y=X \cup Y$, $X \supset Y=\{x \in W \mid \forall y((x R y$ and $y \in X) \Rightarrow y \in Y)\}, \neg X=\{x \in W \mid \forall y(x R y \Rightarrow y \notin X)\}$. Then $\mathbf{B}(\mathbf{W})=<B(W) ; \&, \vee, \supset, \neg, \top>$ is a pseudo-Boolean algebra. 
Lemma 5.1 remains true when $\mathbf{B}(\mathbf{W})$ and $\mathbf{B}\left(\mathbf{W}^{\prime}\right)$ are taken instead of $\mathbf{W}^{+}$and $\mathbf{W}^{\prime+}$ (of course, $\mathbf{W}$ and $\mathbf{W}^{\prime}$ supposed to be reflexive and transitive in this case).

Due to the well-known representation theorems, every modal algebra is embeddable into $\mathbf{W}^{+}$, and every pseudo-Boolean algebra into $\mathbf{B}(\mathbf{W})$ for some suitable frames $\mathbf{W}$. When the given algebra $\mathbf{A}$ is finite then $\mathbf{W}$ is finite and $\mathbf{A}$ is isomorphic to $\mathbf{W}^{+}$(respectively, $\mathbf{B}(\mathbf{W})$ ). It follows that every (modal or superintuitionistic) logic with the Finite Model Property is Kripke-complete.

It is known [22] that the category $K(L)$ of Kripke frames satisfying the modal logic $L$ may be considered as a subcategory of $V(L)$. The p-morphisms of frames correspond to monomorphisms of modal algebras, and cones in frames correspond to homomorphic images.

Let $K$ be a class of frames closed with respect to cones and p-morphisms. Then the amalgamation property for $K^{+}=\left\{\mathbf{W}^{+} \mid \mathbf{W} \in K\right\}$ may be rewritten as follows:

We say that $K$ has the property $A P^{\#}$, whenever for any $\mathbf{W}_{0}, \mathbf{W}_{1}, \mathbf{W}_{2}$ in $K$ and any p-morphisms $\alpha$ from $\mathbf{W}_{1}$ onto $\mathbf{W}_{0}$ and $\beta$ from $\mathbf{W}_{2}$ onto $\mathbf{W}_{0}$ there exist a $\mathbf{W}$ in $K$ and p-morphisms $\varphi$ from $\mathbf{W}$ onto $\mathbf{W}_{1}$ and $\psi$ from $\mathbf{W}$ onto $\mathbf{W}_{2}$ such that $\alpha \varphi=\beta \psi$.

The superamalgamation property for $K^{+}$is equivalent to the following property

$S A P^{\#}$ : For any initial frames $\mathbf{W}_{0}, \mathbf{W}_{1}=\mathbf{W}_{1}{ }^{a}, \mathbf{W}_{2}=\mathbf{W}_{2}{ }^{b}$ in $K$ and any $\mathrm{p}$ morphisms $\alpha$ from $\mathbf{W}_{1}$ onto $\mathbf{W}_{0}$ and $\beta$ from $\mathbf{W}_{2}$ onto $\mathbf{W}_{0}$ such that $\alpha(a)=\beta(b)$ there exist an initial frame $\mathbf{W}=\mathbf{W}^{d}$ in $K$ and p-morphisms $\varphi$ from $\mathbf{W}$ onto $\mathbf{W}_{1}$ and $\psi$ from $\mathbf{W}$ onto $\mathbf{W}_{2}$ such that $\alpha \varphi=\beta \psi$ and $\varphi(d)=a$ and $\psi(d)=b$.

Further, the Joint Embedding Property may be rewritten as follows:

$J E P^{\#}$ : For any $\mathbf{W}_{1}, \mathbf{W}_{2}$ in $K$ there exist a $\mathbf{W}$ in $K$ and p-morphisms $\varphi$ from $\mathbf{W}$ onto $\mathbf{W}_{1}$ and $\psi$ from $\mathbf{W}$ onto $\mathbf{W}_{2}$.

And the $S J E P$ is equivalent to the following property

SJEP\#: For any initial frames $\mathbf{W}_{1}=\mathbf{W}_{1}{ }^{a}, \mathbf{W}_{2}=\mathbf{W}_{2}{ }^{b}$ in $K$ there exist an initial frame $\mathbf{W}=\mathbf{W}^{d}$ in $K$ and p-morphisms $\varphi$ from $\mathbf{W}$ onto $\mathbf{W}_{1}$ and $\psi$ from $\mathbf{W}$ onto $\mathbf{W}_{2}$ such that $\varphi(d)=a$ and $\psi(d)=b$.

We note that for the case of transitive frames the property $S J E P^{\#}$ may be simplified:

For any initial frames $\mathbf{W}_{1}=\mathbf{W}_{1}{ }^{a}, \mathbf{W}_{2}=\mathbf{W}_{2}{ }^{b}$ in $K$ there exist an initial frame $\mathbf{W}=\mathbf{W}^{d}$ in $K$ and p-morphisms $\varphi$ from $\mathbf{W}$ onto $\mathbf{W}_{1}$ and $\psi$ from $\mathbf{W}$ onto $\mathbf{W}_{2}$.

Finally, we rewrite the equivalent $E S^{*}$ of the Beth Property B2. We say that $K$ has the property $E S^{* \#}$ if for any initial frames $\mathbf{W}_{0}$ and $\mathbf{W}_{1}=\mathbf{W}_{1}{ }^{a}=\mathbf{W}_{1}{ }^{b}$ in $K$ and any p-morphisms $\alpha$ and $\beta$ from $\mathbf{W}_{1}$ onto $\mathbf{W}_{0}$ such that $\alpha(a)=\beta(b)$ there exist an initial frame $\mathbf{W}=\mathbf{W}^{d}$ in $K$ and p-morphisms $\varphi$ and $\psi$ from $\mathbf{W}$ onto $\mathbf{W}_{1}$ such that $\alpha \varphi=\beta \psi$ and $\varphi(d)=a$ and $\psi(d)=b$.

For each locally tabular modal logic $L$, every member of the class FGFI $(V(L))$ is a finite modal algebra, so it is isomorphic to $\mathbf{W}^{+}$for a suitable frame $\mathbf{W}$. Therefore, it follows from the theorems of Sections 2 and 3 that $V(L)$ has a property $P$, where $P$ is $S A P, A P, E S^{*}, J E P$ or $S J E P$, if and only if the class $K_{f i n}(L)$ of all finite frames validating $L$ satisfies $P^{\#}$. 
Now let us suppose that a logic $L$ has the Finite Model Property. Then $L$ is complete with respect to the class $K_{f i n}(L)$. In this case, the Hallden-completeness or the PseudoRelevance Property may be reduced to the corresponding properties of $K_{f i n}(L)$. The first part of the following statement follows from [23].

Proposition 5.2. Let a normal modal logic L have the Finite Model Property.

(i) If $K_{\text {fin }}(L)$ has SJEP $P^{\#}$ then $L$ is Hallden-complete.

(ii) If $K_{\text {fin }}(L)$ has JEP $P^{\#}$ then $L$ has the Pseudo-Relevance Property.

On the contrary, the amalgamation properties cannot be reduced to the classes of finite frames.

Proposition 5.3. There exist normal modal logics with the Finite Model Property which satisfy neither CIP nor IPD although $K_{\text {fin }}(L)$ has $S A P^{\#}$.

The first example was given in [10], it is the logic $\mathbf{S 4 . 3 G r z}$ that is complete with respect to the class of finite linearly ordered frames.

Now we consider superintuitionistic logics. It was proved in [7] that every superintuitionistic logic $L$ with the CIP has the Finite Model Property, and the variety $V(L)$ of pseudo-Boolean algebras has the $S A P$ if and only if the class of finite algebras in $V(L)$ satisfies the $A P$. It follows that a superintuitionistic logic $L$ has CIP if and only if $K_{\text {fin }}(L)$ has $A P^{\#}$.

It was mentioned in Section 4 that every superintuitionistic logic has the Beth Property and satisfies the Pseudo-Relevance Principle. As for the Principle of Variable Separation, by analogy with proposition 5.2 , one can prove

Proposition 5.4. Let a superintuitionistic logic L have the Finite Model Property. If $K_{\text {fin }}(L)$ has SJEP\# then L satisfies the Principle of Variable Separation.

The Disjunction Property of a superintuitionistic logic $L$ may be reduced to $K_{\text {fin }}(L)$ as follows. We say that a class $K$ of Kripke frames satisfies $D P^{\#}$ if for any disjoint initial frames $\mathbf{W}_{1}$ and $\mathbf{W}_{2}$ in $K$ there exists an initial frame $\mathbf{W}$ in $K$ such that the direct union $\mathbf{W}_{1} \cup \mathbf{W}_{2}$ is a cone of $\mathbf{W}$.

Proposition 5.5. Let $L$ be a superintuitionistic logic with the Finite Model Property. If $K_{\text {fin }}(L)$ satisfies $D P^{\#}$ then $L$ has the Disjunction Property.

The problem of decidability of the above-mentioned properties was investigated in [1]. In particular, the Hallden Property, DP and PVS are undecidable in the class of superintuitionistic logics; CIP, IPD, HP are undecidable in $N E(\mathbf{K 4})$. At the same time, CIP and IPD are decidable in $N E(\mathbf{S} 4)$ and in the family of all superintuitionistic logics $[7,8]$.

\section{References}

[1] A. Chagrov and M. Zakharyashchev, Undecidability of the disjunction property of propositional logics and other related problems, J. Symbolic Logic 58, 3 (1993), 967-1002. 
[2] M. Dummett and E. Lemmon, Modal logics between S4 and S5, Zeitschr. math. Log. und Grundl. Math. 5 (1959), 250-264.

[3] S. Hallden, On the semantic non-completeness of certain Lewis calculi, J. Symbolic Logic 16 (1951), 127-129.

[4] L. Henkin, J. D. Monk and A. Tarski, Cylindric Algebras, Part II, Amsterdam: NorthHolland, 1985.

[5] Y. Komori, Logics without Craig's interpolation property, Proc. of the Japan Academy, 54 (1978), 46-48.

[6] G. Kreisel, Explicit definability in intuitionistic logic, J. Symbolic Logic, 25 (1960), 389-390.

[7] L. L. Maksimova, Craig's Theorem in Superintuitionistic Logics and Amalgamable Varieties of Pseudo-Boolean Algebras, Algebra i Logika, 16, no. 6 (1977), 643-681.

[8] L. L. MaKsimova, Interpolation theorems in modal logics and amalgamated varieties of topoboolean algebras, Algebra i Logika, 18, No. 5, 556-586 (1979).

[9] L. Maksimova, Interpolation properties of superintuitionistic logics, Studia Logica 38 (1979), 419-428.

[10] L. Maksimova, Failure of interpolation property in modal companions of Dummett's logic, Algebra i Logika, 21, no. 6 (1982), 690-694.

[11] L. Maksimova, On maximal intermediate logics with the disjunction property, Studia Logica, 45, no. 1 (1986), 69-75.

[12] L. Maksimova, Amalgamation and Interpolation in Normal Modal Logics, Studia Logica, 50, 3/4 (1991), 457-471.

[13] L. L. Maksimova, Modal logics and varieties of modal algebras: the Beth properties, interpolation and amalgamation, Algebra i Logika 31, 2 (1992), 145-166.

[14] L. Maksimova, Definability and Interpolation in Classical Modal Logics, Contemporary Mathematics, 131, 1992 (part 3), 583-599.

[15] L. L. Maksimova, An Analog of Beth's Theorem in Normal Extensions of the Modal Logic K4, Sibirskii Matematicheskii Zhurnal, 33, no. 6 (1992), 118-130.

[16] L. Maksimova, On variable separation in Modal and Superintuitionistic Logics, Studia Logica, 55 (1995), 99-112.

[17] L. L. Maksimova and V. V. Rybakov, On the lattice of normal modal logics, Algebra i Logika 13, 2 (1974), 188-216.

[18] H. Rasiowa and R. Sikorski, The Mathematics of Metamathematics, PWN, Warsaw, 1963.

[19] W. Rautenberg, Klassische und nicht-klassische Aussagenlogik, Wiesbaden, Vieweg, Braunschweig, 1979.

[20] K. Segerberg, An Essay in Classical Modal Logics, Uppsala University, Uppsala, 1971.

[21] N.-Y. SuZUKI, Intermediate logics characterized by a class of algebraic frames with infinite individual domain, Bull. Section of Logic 18, 2 (1989), 63-71.

[22] S. K. Thomason, Categories of frames for modal logic, J. Symbolic Logic, 40, no. 3 (1975), 439-442.

[23] J. F. A. K. van Benthem and I. L. Humberstone, Hallden-completeness by gluing of Kripke-frames, Notre Dame J. of Formal Logic, 24 (1983), 426-430.

[24] A. Wroński, Remarks on Hallden-completeness of modal and intermediate logics, Bull. Section of Logic 5, 4 (1976), 126-129. 